\title{
Change in Entropy of Spinning Black Holes Due to Corresponding Change in Mass in AGN
}

\author{
Anuradha Kumari' ${ }^{1}$, Supriya Kumari', Dipo Mahto² \\ ${ }^{1}$ Department of Physics, T. M. B. University, Bhagalpur, India \\ ${ }^{2}$ Department of Physics, Marwari College, T. M. B. University, Bhagalpur, India \\ Email: dipomahto@hotmail.com
}

How to cite this paper: Kumari, A., Kumari, S. and Mahto, D. (2018) Change in Entropy of Spinning Black Holes Due to Corresponding Change in Mass in AGN. Journal of Applied Mathematics and Physics, 6, 1686-1691.

https://doi.org/10.4236/jamp.2018.68144

Received: April 5, 2018

Accepted: August 19, 2018

Published: August 22, 2018

Copyright (c) 2018 by authors and Scientific Research Publishing Inc. This work is licensed under the Creative Commons Attribution International License (CC BY 4.0).

http://creativecommons.org/licenses/by/4.0/

\begin{abstract}
The present paper discusses the change in entropy of spinning black holes due to change in mass for AGN black holes on the basis of the model as proposed in the case of XRBs under condition that the black holes spin with unit spinning parameter and angular momentum and this model gives slightly some different nature of the super-massive spinning black holes in AGN as specified for XRBs.
\end{abstract}

\section{Keywords}

Entropy Change, Angular Velocity, Spin Parameter

\section{Introduction}

According to the theory of relativity, a black hole is the solution of Einstein's gravitational field equations in the absence of matter that describes the space time around a gravitationally collapsed star and its gravitational pull too strong that even light cannot escape from it [1], while classically, the black holes are perfect absorbers and do not emit anything; their temperature is absolute zero. However, in quantum theory black holes emit Hawking radiation with a perfect thermal spectrum [2] [3]. Mahto et al. proposed a model for the change in entropy of non-spinning black holes w.r.t. change in the event horizon of black holes using the first law of black hole Physics [4]. Mahto, D., Kumari, A. and Singh, K.M. have shown that there is no any change in the energy and entropy of black holes having zero spin and leading the second laws of thermodynamics ( $d S \geq 0$ ) [5]. Mahto, D. and Kumari, A. proposed a model for the change in entropy of spinning black holes due to change in mass using first law of black hole mechanics for unit spinning parameter and angular momentum and also calculated their values for different spinning black holes in XRBs [6]. 
In the present paper, we have applied the same model to discuss the change in entropy of spinning black holes due to change in mass for AGN black holes and used this model to calculate their values for different test spinning black holes in AGN.

\section{Theoretical Model Used}

The change in entropy of spinning black holes due to change in mass using first law of black hole mechanics for unit spinning parameter and angular momentum is given by the following equation [6].

$$
\left|\frac{\delta S}{\delta M}\right|=24 \pi M^{2}
$$

\section{Data in the Support for Mass of Super-Massive Black Holes}

The super massive black holes in Galactic nuclei have their masses range estimated is $\mathrm{M} \sim 10^{6}-10^{9.5} M_{\odot}$ [7]. Masses of "central dark object" have been estimated in about forty cases, using stellar dynamics, emission lines of orbiting gas and, most accurately, using water masers. They range from $\sim 2 \times 10^{6} M_{\odot} t o \sim 3 \times 10^{9} M_{\odot}$ and, in many cases, the compactness is sufficient to rule out star clusters with confidence [8]. Most detected super massive black holes are in the $10^{8} \leq M_{.} \leq 10^{9} M_{\odot}$ range, there are no detections below $10^{6} M_{\odot}$ (the "building block" range) or above $10^{10} M_{\odot}$ (the brightest quasar range), and even the $10^{6} \leq M_{\bullet} \leq 10^{7} M_{\odot}$ range is very poorly sampled [9].

\section{Data in the Support for the Mass of Sun}

$$
\text { Mass of } \operatorname{sun}\left(M_{\odot}\right)=1.99 \times 10^{30} \mathrm{~kg},[7]
$$

All the above primary data are in terms of solar mass in AGN black holes and we have used the model represented by the Equation (1) to derive the secondary data in terms of entropy change and calculated their values of different test spinning black holes of spin parameter $\mathrm{a}^{\star}=1$ and unit angular velocity in AGN. These are listed in the following Table 1 .

\section{Results and Discussion}

The present work is extended part of the model for the change in entropy of spinning black holes due to change in mass in XRBs black holes expressed by the Equation (1) and it is applied for different test black holes in AGN [6].

Using the model as proposed by the Equation (1), we have plotted the graph between the mass of spinning black holes in terms of $10^{7}$ solar mass and the magnitude of the change in entropy with change in mass of spinning black holes with the help of Table 1, which is shown in Figure 1. This shows that the magnitude of change in entropy with change in mass increases with the increasing the mass of different test spinning black holes in AGN. 
The graph plotted between the mass of spinning black holes and the magnitude of the change in entropy with change in mass of spinning black holes is approximately in straight line as shown in Figure 1. The straight line nature of the graph gives the validity of present model. In fact, there is some slightly deviation in the nature of straight line of graph for the black holes lying the masses between $4 \times 10^{8}$ to $6 \times 10^{8}$ solar mass. When we observed the data of mass of the black holes, we see that these masses are intermediate masses of black holes among AGNs. In this region, there is some slightly non-uniform variation in the entropy change with change in mass of spinning black holes.

In spite of the above merits, this graph is not able to provide too much clear description regarding the change in entropy with change in mass of spinning black holes, because the mass of the black holes ranging from 0.1 solar mass to 30 solar mass is closely packed so that distinct vision for the entropy change with mass is difficult to view.

To remove the above deficiency, when we have used the logarithmic scale to plot the graph 2 using the same data as plotted the graph 1, the change in entropy with change in mass of spinning black holes ranging from 0.1 solar mass to 30 solar mass is not closely packed and hence the distinct vision for the entropy change with mass is view clearly. Figure 2 indicates that the change in entropy with change in mass of spinning black holes remains constant in ranging from 0.1 solar mass to 10 solar mass and after this, an abrupt change in entropy takes place with change in mass of spinning black holes.

\section{Conclusions}

1) The magnitude of the change in entropy with change in mass of spinning black holes is directly proportional to the square of their masses.

2) The magnitude of the change in entropy with change in mass of uncharged spinning black holes is essentially the function for AGN black holes.

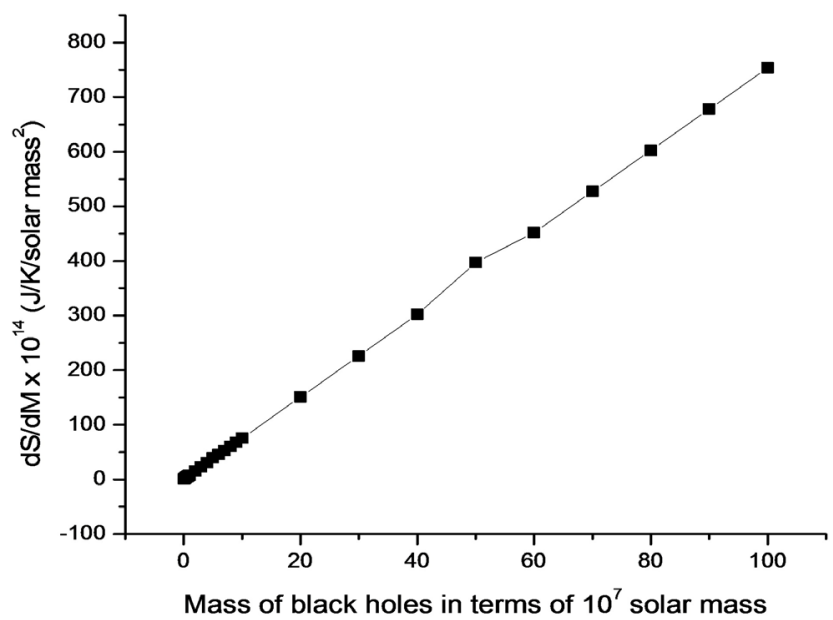

Figure 1. The graph plotted between the mass of black holes in terms of $10^{7}$ solar mass and their corresponding change in entropy relative to the change in mass for AGN. 


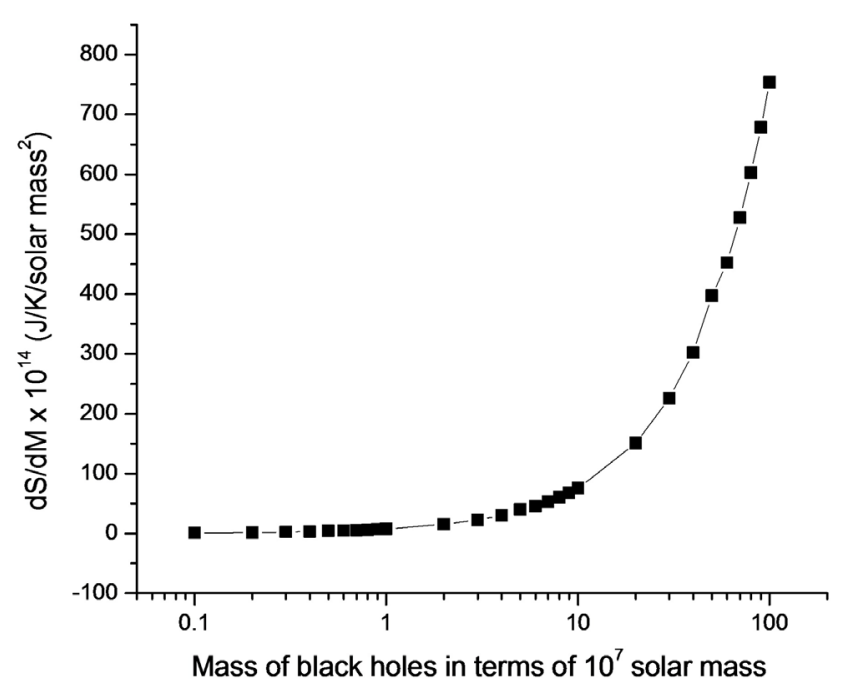

Figure 2. The graph plotted between the mass of black holes in terms of $10^{7}$ solar mass and their corresponding change in entropy relative to the change in mass for AGN on logarithmic scale.

Table 1. Change in entropy of spinning black holes due to corresponding change in mass in AGN.

\begin{tabular}{|c|c|c|c|c|c|}
\hline $\begin{array}{l}\text { S. } \\
\text { No. }\end{array}$ & $\begin{array}{c}\text { Mass of } B H_{s}(\mathrm{M}) \\
\text { in solar masses }\end{array}$ & $\begin{array}{l}\text { Mass of } \mathrm{BHs} \text { in } \\
\text { terms of } 10^{7} \mathrm{M}_{\odot}\end{array}$ & $a^{*}$ & $\Omega$ & $\left|\frac{\delta S}{\delta M}\right|$ \\
\hline 1 & $1 \times 10^{6} \mathrm{M}_{\odot}$ & $0.1 \mathrm{M}_{\odot}$ & 1 & 1 & $0.7536 \times 10^{14}$ \\
\hline 2 & $2 \times 10^{6} \mathrm{M}_{\odot}$ & $0.2 \mathrm{M}_{\odot}$ & 1 & 1 & $1.507 \times 10^{14}$ \\
\hline 3 & $3 \times 10^{6} \mathrm{M}_{\odot}$ & $0.3 \mathrm{M}_{\odot}$ & 1 & 1 & $2.260 \times 10^{14}$ \\
\hline 4 & $4 \times 10^{6} \mathrm{M}_{\odot}$ & $0.4 \mathrm{M}_{\odot}$ & 1 & 1 & $3.024 \times 10^{14}$ \\
\hline 5 & $5 \times 10^{6} \mathrm{M}_{\odot}$ & $0.5 \mathrm{M}_{\odot}$ & 1 & 1 & $3.968 \times 10^{14}$ \\
\hline 6 & $6 \times 10^{6} \mathrm{M}_{\odot}$ & $0.6 \mathrm{M}_{\odot}$ & 1 & 1 & $4.521 \times 10^{14}$ \\
\hline 7 & $7 \times 10^{6} \mathrm{M}_{\odot}$ & $0.7 \mathrm{M}_{\odot}$ & 1 & 1 & $5.275 \times 10^{14}$ \\
\hline 8 & $8 \times 10^{6} \mathrm{M}_{\odot}$ & $0.8 \mathrm{M}_{\odot}$ & 1 & 1 & $6.028 \times 10^{14}$ \\
\hline 9 & $9 \times 10^{6} \mathrm{M}_{\odot}$ & $0.9 \mathrm{M}_{\odot}$ & 1 & 1 & $6.782 \times 10^{14}$ \\
\hline 10 & $1 \times 10^{7} \mathrm{M}_{\odot}$ & $1 \mathrm{M}_{\odot}$ & 1 & 1 & $7.5360 \times 10^{14}$ \\
\hline 11 & $2 \times 10^{7} \mathrm{M}_{\odot}$ & $2 \mathrm{M}_{\odot}$ & 1 & 1 & $15.075 \times 10^{14}$ \\
\hline 12 & $3 \times 10^{7} \mathrm{M}_{\odot}$ & $3 \mathrm{M}_{\odot}$ & 1 & 1 & $22.603 \times 10^{14}$ \\
\hline 13 & $4 \times 10^{7} \mathrm{M}_{\odot}$ & $4 \mathrm{M}_{\odot}$ & 1 & 1 & $30.240 \times 10^{14}$ \\
\hline 14 & $5 \times 10^{7} \mathrm{M}_{\odot}$ & $5 \mathrm{M}_{\odot}$ & 1 & 1 & $39.680 \times 10^{14}$ \\
\hline 15 & $6 \times 10^{7} \mathrm{M}_{\odot}$ & $6 \mathrm{M}_{\odot}$ & 1 & 1 & $45.216 \times 10^{14}$ \\
\hline 16 & $7 \times 10^{7} \mathrm{M}_{\odot}$ & $7 \mathrm{M}_{\odot}$ & 1 & 1 & $52.752 \times 10^{14}$ \\
\hline 17 & $8 \times 10^{7} \mathrm{M}_{\odot}$ & $8 \mathrm{M}_{\odot}$ & 1 & 1 & $60.288 \times 10^{14}$ \\
\hline 18 & $9 \times 10^{7} \mathrm{M}_{\odot}$ & $9 \mathrm{M}_{\odot}$ & 1 & 1 & $67.824 \times 10^{14}$ \\
\hline 19 & $1 \times 10^{8} \mathrm{M}_{\odot}$ & $10 \mathrm{M}_{\odot}$ & 1 & 1 & $75.360 \times 10^{14}$ \\
\hline 20 & $2 \times 10^{8} \mathrm{M}_{\odot}$ & $20 \mathrm{M}_{\odot}$ & 1 & 1 & $150.750 \times 10^{14}$ \\
\hline
\end{tabular}




\begin{tabular}{cccccc}
\multicolumn{7}{l}{ Continued } \\
\hline 21 & $3 \times 10^{8} \mathrm{M}_{\odot}$ & $30 \mathrm{M}_{\odot}$ & 1 & 1 & $226.030 \times 10^{14}$ \\
22 & $4 \times 10^{8} \mathrm{M}_{\odot}$ & $40 \mathrm{M}_{\odot}$ & 1 & 1 & $302.400 \times 10^{14}$ \\
23 & $5 \times 10^{8} \mathrm{M}_{\odot}$ & $50 \mathrm{M}_{\odot}$ & 1 & 1 & $396.800 \times 10^{14}$ \\
24 & $6 \times 10^{8} \mathrm{M}_{\odot}$ & $60 \mathrm{M}_{\odot}$ & 1 & 1 & $452.160 \times 10^{14}$ \\
25 & $7 \times 10^{8} \mathrm{M}_{\odot}$ & $70 \mathrm{M}_{\odot}$ & 1 & 1 & $527.520 \times 10^{14}$ \\
26 & $8 \times 10^{8} \mathrm{M}_{\odot}$ & $80 \mathrm{M}_{\odot}$ & 1 & 1 & $602.880 \times 10^{14}$ \\
27 & $9 \times 10^{8} \mathrm{M}_{\odot}$ & $90 \mathrm{M}_{\odot}$ & 1 & 1 & $678.240 \times 10^{14}$ \\
28 & $1 \times 10^{9} \mathrm{M}_{\odot}$ & $100 \mathrm{M}_{\odot}$ & 1 & 1 & $753.600 \times 10^{14}$ \\
\hline
\end{tabular}

3) On increasing the mass of spinning black holes, their corresponding magnitude of change in their entropy with change in mass increases linearly, but some little deviation is between the intermediate mass with spin parameter $\mathrm{a}^{*}=$ 1 and $\Omega=1$.

\section{Acknowledgements}

The authors are very grateful to reviewers to find out the errors in the original manuscript and providing the constructive suggestions. This paper is devoted in memory of great scientist Stephen Hawking (March 14, 2018). The authors are also obliged to UGC to give the financial support under the scheme of Minor Research Project in Science having No. PSB-011/15-16, (ERO) ID No. BBH1-021, S. No. 227847.

\section{Conflicts of Interest}

The authors declare no conflicts of interest regarding the publication of this paper.

\section{References}

[1] Dabholkar, A. (2006) Black Hole Entropy in String Theory: A Window into the Quantum Structure of Gravity. Current Science, 89, 2054-2063. https://doi.org/10.1142/9789812772718_0004

[2] Bardeen, J.M., Carter, B. and Hawking, S.W. (1973) The Four Laws of Black Hole Mechanics. Communications in Mathematical Physics, 31, 161-170. https://doi.org/10.1007/BF01645742

[3] Wald, R.M. (2001) The Thermodynamics of Black Holes. Living Reviews in Relativity, 4, 6. https://www.springer.com/cn/livingreviews https://doi.org/10.12942/lrr-2001-6

[4] Mahto, D., Prakash, V., Prasad, U. and Singh, B.K. (2013) Change in Entropy of Non-Spinning Black Holes w.r.t. the Radius of Event Horizon in XRBs. Astrophysics and Space Science, 343, 153-159. https://doi.org/10.1007/s10509-012-1219-y

[5] Mahto, D., Kumari, A. and Singh, K.M. (2013) Energy and Entropy Change of Spinning Black Holes. International Journal of Engineering and Innovative Technology, 3, 270-273. https://doi.org/10.18052/www.scipress.com/ILCPA.23.12 
[6] Mahto, D. and Kumari, A. (2018) Change in Entropy of Spinning Black Holes Due to Corresponding Change in Mass in XRBs. International Journal of Astronomy and Astrophysics, 8, 171-177. https://doi.org/10.4236/ijaa.2018.82012

[7] Narayan, R. (2005) Black Holes in Astrophysics. New Journal Physics, 7, 1-31. https://doi.org/10.1088/1367-2630/7/1/199

[8] Ferrarese, L. and Ford, H. (2005) Super Massive Black Holes in Galactic Nuclei: Past Present \& Future Research. Space Science Reviews, 116, 523-624. https://doi.org/10.1007/s11214-005-3947-6

[9] Blandford, R.D. (1999) Recent Results on Active Galactic Nuclei. Astrophysics and Space Science, 261, 245-252. https://doi.org/10.1023/A:1002093710287 